AIAA-99-4120

\title{
DYNAMICS AND CONTROL OF A NOVEL RADIO TELESCOPE ANTENNA
}

\author{
Meyer Nahon* \\ Space and Subsea Robotics Laboratory \\ Department of Mechanical Engineering, University of Victoria \\ P.O. Box 3055, Victoria, B.C., Canada V8W 3P6
}

\begin{abstract}
This paper presents a mathematical and computer model of the triple-tethered aerostat proposed to support the receiver in a very large radio telescope antenna. The dynamics model considers the system as a set of three (or more) tethers, attached at fixed points on the ground, which come together at a spherical confluence point in which the receiver is located. Also attached to the confluence point is a leash tied to an aerostat overhead. The aerostat provides the necessary lifting force to keep the system aloft. At the ground attachment points, winches pull on the tethers to maintain the confluence point at its desired location. To investigate the performance of this system, we assembled a numerical model of its equations of motion. The tethers and leash were discretized into a number of elements using a lumped mass approach. The effects of cable stiffness, internal damping, gravity and aerodynamic drag as well as winds and turbulence were included in the model. The spherical confluence point and aerostat were modeled to include the effects of aerodynamic forces, as well as gravity and buoyancy. A controller was then developed to control the tether lengths in response to errors in the receiver position from a desired location. The complete system of almost 200 simultaneous first-order differential equations was formulated and solved numerically using a fourth-order Runge-Kutta integration scheme. Numerical experiments on this system indicate that the system can be accurately controlled in the presence of disturbances and that the concept warrants further study.
\end{abstract}

\section{Introduction}

Radio astronomers from around the world ${ }^{1}$ have focussed recently on the need for a new radio telescope that would enable the direct observation of the formation and evolution of galaxies from gases in the universe. ${ }^{2}$ To do this, requires an increase in sensitivity of roughly two orders of magnitude over existing radio telescope arrays---to a collecting area of $10^{6} \mathrm{~m}^{2}$ and is thus dubbed the Square Kilometer Array (SKA). One such conceptual design originates from the National Research Council of Canada's Herzberg Institute of Astrophysics and consists of an array of about 30 very large antennas, each of about 200-300 m diameter. The proposed novel antenna design, ${ }^{3}$ a single one of which is depicted in Figure 1, is called the Large Adaptive Reflector (LAR).

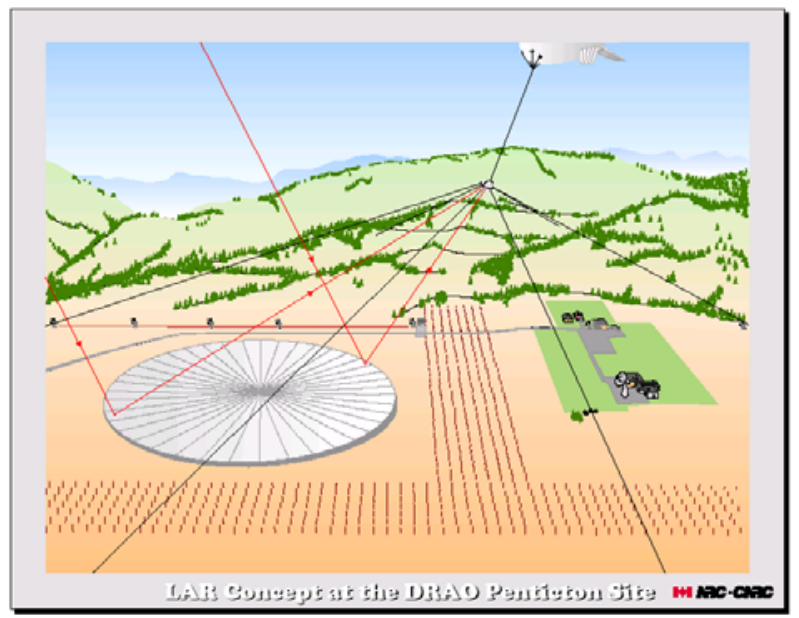

Figure 1. The Proposed Large Adaptive Reflector ${ }^{8}$

The LAR design is based on the premise that building a large scale steerable radio antenna will require new concepts in the design of structures to achieve the required strength and stiffness at a reasonable cost. The focus of each reflector will consist of a receiver held aloft at an altitude of $\mathrm{R}=500 \mathrm{~m}$ by a tethered aerostat. To fulfil its function, the receiver must be positioned accurately at points on the surface of a hemisphere of radius $\mathrm{R}$, centered at the center of the reflector. This will be accomplished by adjusting the tether lengths. It

* Associate Professor, Senior Member AIAA

Copyright (C) 1999 The American Institute of Aeronautics and Astronautics, Inc.

All rights reserved. 
is also envisioned that the variable-length tethers will allow some measure of control of the receiver position in response to disturbances such as wind gusts.

A key issue which will determine whether the LAR design is feasible is that of positioning accuracy of the receiver. Among the questions which must be answered are: how much will typical winds and gusts disturb the receiver position? and how effective might the tether winches be at overcoming these effects ?

Tethered and moored aerostat systems have received limited attention in the literature ${ }^{4,5}$ and these have usually consisted of a large streamlined aerostat constrained by a single tether. These studies have focussed on the open-loop (uncontrolled) behavior of such systems. One key issue in modeling these systems is that of determination of the aerodynamic characteristics of the aerostat. ${ }^{6,7}$ The aerostat design for LAR has not yet been finalized and may be spherical or streamlined. Some prior work at the National Research Council of $\mathrm{Canada}^{8}$ dealt with a triple-tethered aerostat system, but focussed primarily on its static or steadystate performance.

Since a prototype would be prohibitively expensive to construct at this stage, a mathematical model and a computer simulation were assembled to study the dynamics and control of the system. These may be used for proof of concept studies and as design tools. A model of the planetary boundary layer mean wind and turbulence is implemented to investigate the effect of these disturbances on the system. A range of typical strong winds from a range of azimuthal directions are used and their effect is quantified with the receiver at a variety of zenith and azimuth angles (i.e. different receiver positions on its hemispherical trajectory). The effectiveness of the tether winches at controlling the receiver position is then evaluated using a simple controller consisting of independent PID loops on each tether.

\section{Model Overview}

The complete tethered aerostat system is shown diagrammatically in 2-D in Figure 2. The model consists of the following elements: (a) an aerostat of 40 $\mathrm{KN}$ gross lift, (b) a leash joining the aerostat to the receiver below it, (c) the receiver which is enclosed in a spherical shell, and (d) three elastic tethers from the ground to the common attachment point at the receiver. The aerostat is modeled as a single mass at the upper node of the leash, subject to buoyancy, aerodynamic drag (generated by winds and gusts), and gravity. Added mass of the aerostat is included, since it is large. The leash and tethers are modeled as a series of discrete cable elements connected by frictionless revolute joints.
This effectively neglects the effects of bending stiffness since the dominant forces are due to tension. The mass of each element is lumped into its respective end nodes. The payload is modeled as a spherical point mass subject to gravity and aerodynamic drag.

The forces in the cable model are broken down into two types: internal and external forces. Forces generated within the cable are called internal forces and are due to axial stiffness and internal damping. Forces exerted on the rope by the environment are external forces, and consist of the aerodynamic drag and gravitational forces. The equations for internal forces and the drag forces are developed in an elemental bodyfixed frame, while the equations for the gravitational forces are developed in an inertial frame. The motion equations are written in the inertial frame, and thus all forces must be transformed into that frame prior to inclusion in these equations.

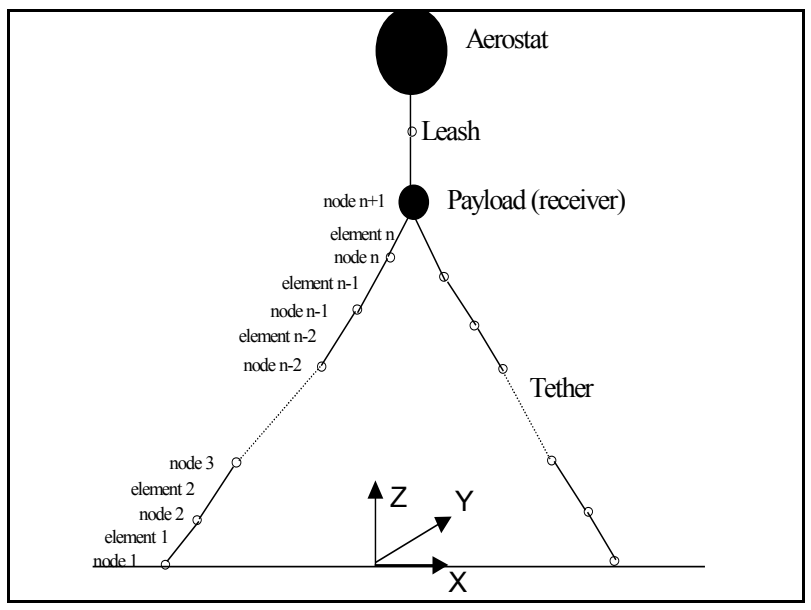

Figure 2. Discrete Implementation of Tethered Aerostat System

\section{Cable Model}

Two distinct types of orthogonal reference frame are used in the development of the mathematical model, an inertial reference frame and a series of body fixed reference frame placed at the nodes along the cables. The inertial reference frame $(X, Y, Z)$ is defined with its origin located at the ground, at the center of the three tether attachment points. The $X$ axis is in the horizontal plane and directed from the origin of the inertial frame to the base attachment point of Tether $\# 1$. The $Z$ axis is vertical and positive upward, as shown in Fig. 2. Finally, the $Y$ axis completes a right-handed coordinate system. The elemental body-fixed frame $\left(p_{1}, p_{2}, q\right)$, is defined relative to each cable element, where $p_{1}$ and $p_{2}$ are the local normal and binormal directions, while $q$ is the local tangent to the cable. 
The solution of continuous cable models subject to general external forces is usually not possible and it is therefore considered more expedient to use a discrete model. The present work applies a lumped mass model with which we have a great deal of experience. This model has been validated for a variety of underwater systems with excellent agreement with in-field measurements. ${ }^{9,10,11}$

\section{$\underline{\text { Kinematics }}$}

The position of each node is described with respect to an inertial reference frame, by a three-component vector $\mathbf{r}^{i}=\left[\begin{array}{llll}r_{X}^{i} & r_{Y}^{i} & r_{Z}^{i}\end{array}\right]^{\mathrm{T}}$. Each cable element is considered to be a straight elastic element, subject to forces at its end points. This method of modeling allows each cable element to possess distinct properties, such as density and stiffness.

The orientation of each cable element is represented using a Z-Y-X $(\psi, \theta, \phi)$ Euler angle set. These three successive rotations align the inertial frame with the $i$-th body frame. Since the torsion of the cable is not included in the model, the $\psi$ rotation about the inertial $\mathrm{Z}$ axis is constrained to zero. The resulting orthogonal rotation matrix which transforms vectors from the bodyfixed frame of the $i$-th element to the inertial frame is denoted as ${ }^{10}$

$$
\boldsymbol{R}_{I B}^{i}=\left[\begin{array}{ccc}
\cos \theta^{i} & \sin \theta^{i} \sin \phi^{i} & \sin \theta^{i} \cos \phi^{i} \\
0 & \cos \phi^{i} & -\sin \phi^{i} \\
-\sin \theta^{i} & \cos \theta^{i} \sin \phi^{i} & \cos \theta^{i} \cos \phi^{i}
\end{array}\right]
$$

The Euler angles can be calculated from the coordinates of the appropriate nodal end points. For example, consider the $i$-th cable element which is bounded by nodes $i$ and $i-1$, shown in Fig. 3.

When expressed in the body frame, the only non-zero component of the vector $\mathbf{r}^{i}-\mathbf{r}^{i-1}$ is in the tangential direction. In component form, we have:

$$
\boldsymbol{R}_{I B}^{i}\left\{\begin{array}{c}
0 \\
0 \\
l^{i}
\end{array}\right\}=\left\{\begin{array}{c}
r_{X}^{i}-r_{X}^{i-1} \\
r_{Y}^{i}-r_{Y}^{i-1} \\
r_{Z}^{i}-r_{Z}^{i-1}
\end{array}\right\}
$$

where $l^{i}$, the length of the $i$-th element, is given by

$$
l^{i}=\sqrt{\left(r_{X}^{i}-r_{X}^{i-1}\right)^{2}+\left(r_{Y}^{i}-r_{Y}^{i-1}\right)^{2}+\left(r_{Z}^{i}-r_{Z}^{i-1}\right)^{2}}
$$

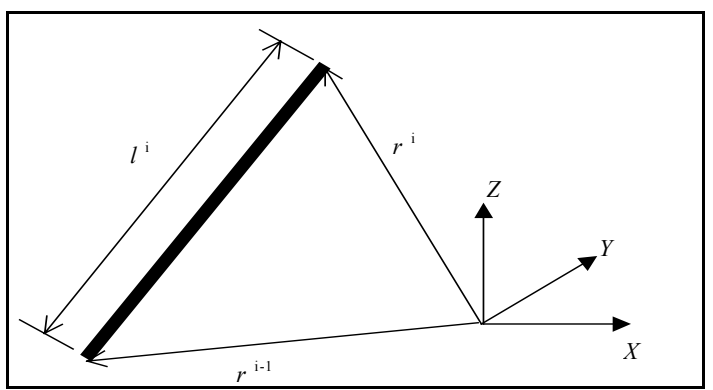

Figure 3. The $i$-th Cable Element.

Substitution of (1) into (2) results in the following set of equations $^{10}$

$$
\begin{aligned}
& l^{i} \sin \theta^{i} \cos \phi^{i}=\left(r_{X}^{i}-r_{X}^{i-1}\right) \\
& -l^{i} \sin \phi^{i}=\left(r_{Y}^{i}-r_{Y}^{i-1}\right) \\
& l^{i} \cos \theta^{i} \cos \phi^{i}=\left(r_{Z}^{i}-r_{Z}^{i-1}\right)
\end{aligned}
$$

which can be combined to yield

$$
\theta^{i}=\operatorname{atan} 2\left(r_{X}^{i}-r_{X}^{i-1}, r_{Z}^{i}-r_{Z}^{i-1}\right)
$$

The solution for $\phi^{i}$ can then be found in either of two ways, depending on which is more numerically stable:

$$
\begin{aligned}
& \phi^{i}=\operatorname{atan} 2\left[-\left(r_{Y}^{i}-r_{Y}^{i-1}\right), \frac{\left(r_{Z}^{i}-r_{Z}^{i-1}\right)}{\cos \theta^{i}}\right] \quad \text { if } \cos \theta \geq \sin \theta^{i} \\
& \phi^{i}=\operatorname{atan} 2\left[-\left(r_{Y}^{i}-r_{Y}^{i-1}\right), \frac{\left(r_{X}^{i}-r_{X}^{i-1}\right)}{\sin \theta^{i}}\right] \quad \text { if } \cos \theta<\sin \theta^{i}
\end{aligned}
$$

Thus, (5) and (6) are our desired relations which allow us to find an element's Euler angles from its nodal coordinates.

\section{Internal Forces}

The internal forces acting within an element are due to its viscoelastic properties. These are represented schematically in Fig. 4.

The tension in the cable due to its structural stiffness is considered to act only in the tangential direction and is modeled by a linear function relating tension and strain

$$
T_{q}^{i}=A E \varepsilon, \quad \varepsilon=\frac{l^{i}-l_{u}^{i}}{l_{u}^{i}}
$$

where $l_{u}^{i}$ is the unstretched length of the $i$-th element, $A$ is its cross sectional area, $E$ is the effective Young's modulus of the cable, and $\varepsilon$ is the strain. The tension is set to zero if the strain becomes negative. However, this condition was never reached in the cases investigated since the aerostat buoyancy is very large in relation to the weight of the system aloft. 


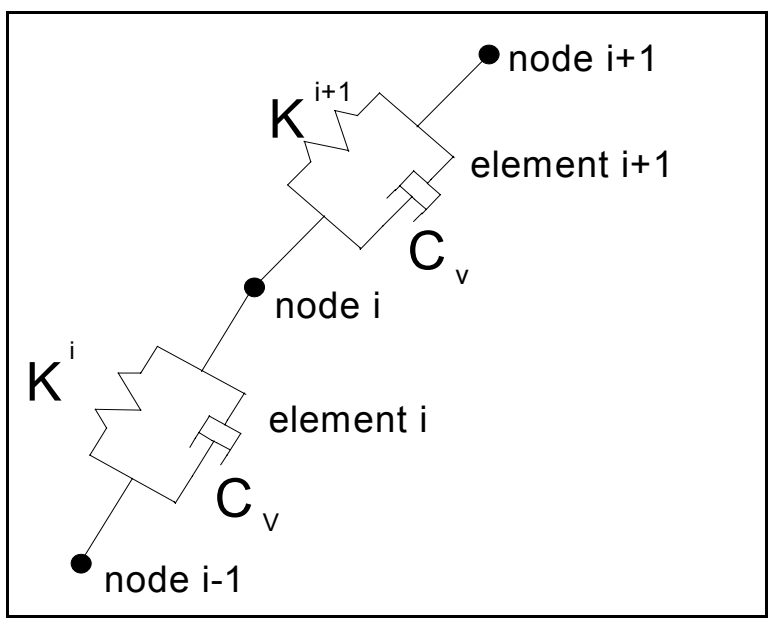

Figure 4. Schematic Representation of the Internal Forces

The friction between the braids of the cable tends to create a damping effect. This effect is assumed to be linear with the following relationship between strain rate and damping force

$$
P_{q}^{i}=C_{v}\left(v_{q}^{i}-v_{q}^{i-1}\right)
$$

where $v_{q}^{i}$ is the velocity of the $i$-th node in the tangential direction.

\section{External Forces}

The external forces acting on the cable element are those due to aerodynamic drag and gravity. The drag forces acting on the cable element can be calculated according to ${ }^{10}$

$$
\begin{aligned}
& D_{p 1}^{i}=-\left(\frac{1}{2} \rho C_{d} d_{c} l_{u}^{i}\right) f_{p}\left|\mathbf{v}^{i}\right|^{2} \frac{v_{p 1}^{i}}{\sqrt{\left(v_{p 1}^{i}\right)^{2}+\left(v_{p 2}^{i}\right)^{2}}} \\
& D_{p 2}^{i}=-\left(\frac{1}{2} \rho C_{d} d_{c} l_{u}^{i}\right) f_{p}\left|\mathbf{v}^{i}\right|^{2} \frac{v_{p 2}^{i}}{\sqrt{\left(v_{p 1}^{i}\right)^{2}+\left(v_{p 2}^{i}\right)^{2}}} \\
& D_{q}^{i}=-\left(\frac{1}{2} \rho C_{d} d_{c} l_{u}^{i}\right) f_{q}\left|\mathbf{v}^{i}\right|^{2}
\end{aligned}
$$

where $\mathbf{D}^{i}$ is the aerodynamic drag force represented in the body-fixed frame with components $D_{p 1}^{i}, D_{p 2}^{i}$ and $D_{q}^{i} ; \rho$ is the local density of air; $d_{c}$ is the cable diameter; $C_{d}$ is the normal drag coefficient of the cable; and $\mathbf{v}^{i}$ is the local velocity of the geometric center of the $i$-th cable element with respect to the surrounding air, with components $v_{p 1}^{i}, v_{p 2}^{i}$, and $v_{q}^{i}$. These velocities must account not only for the motion of the cable element, but also the motion of the surrounding air (to be discussed in a later section). In each of the above equations, the drag coefficient is modified by an appropriate loading function, $f_{p}$ or $f_{q}$ which are functions of $\eta$, the relative angle between the $i$-th element and the incident fluid flow. These loading functions account for the nonlinear breakup of drag between the normal and tangential directions. Driscoll and Nahon ${ }^{9}$ surveyed several papers ${ }^{12,13,14}$ which studied marine cable in a variety of towing conditions and arrived at the following synthesis of these models

$$
\begin{aligned}
f_{p}= & 0.5-0.1 \cos \eta+0.1 \sin \eta-0.4 \cos 2 \eta-0.011 \sin 2 \eta \\
f_{q}= & 0.01\left(2.008-0.3858 \eta+1.9159 \eta^{2}-4.16147 \eta^{3}\right. \\
& \left.+3.5064 \eta^{4}-1.187299 \eta^{5}\right)
\end{aligned}
$$

where $\eta$ is expressed in radians and $0 \leq \eta \leq \pi / 2$. The relative velocity of the flow over the geometric center of a particular cable element is found by averaging the relative velocities of its two end nodes, where the relative velocity of the air over node $i$ is a function of the air velocity as well as the velocity of the node: $\left\|\mathbf{v}^{i}\right\|=\sqrt{\left(U_{X}^{i}-\frac{d r_{X}^{i}}{d t}\right)^{2}+\left(U_{Y}^{i}-\frac{d r_{Y}^{i}}{d t}\right)^{2}+\left(U_{Z}^{i}-\frac{d r_{Z}^{i}}{d t}\right)^{2}}$

where $U_{X}^{i}, U_{Y}^{i}$, and $U_{Z}^{i}$ are the local air velocity components at node $i$ due to the mean wind and air turbulence. Once the drag for elements $i$ and $i+1$ are calculated, half of each value is applied to the $i$-th node which joins the two elements.

Finally, the equation for the net gravitational force acting on a cable element in the inertial frame is:

$$
W^{i}=\rho_{c} \frac{\pi d_{c}^{2}}{4} l_{u}^{i} g
$$

where $\rho_{c}$ is the density of material of the element; and $g$ is the acceleration due to gravity.

\section{Aerostat and Payload Models}

In the present work, the aerostat is assumed to be spherical. Although a streamlined aerostat of equivalent lift would have significantly lower drag, and therefore be less affected by the local wind, it would also be much more expensive to purchase and operate. It was therefore preferred to first investigate the feasibility of using a spherical aerostat, while leaving the streamlined aerostat as a future backup option, should the spherical aerostat not be controllable to the required precision.

The payload located at the upper confluence point of the three tethers contains the antenna receiver and is assumed to be spherical in shape. Thus, for both the aerostat and the payload, we can write

$$
D=\frac{1}{2} \rho C_{d} \frac{\pi d^{2}}{4}|\mathbf{v}|^{2}
$$


where $D$ is the aerodynamic drag force; $d$ is the aerostat or payload diameter; $C_{d}$ is the normal drag coefficient; and $\mathbf{v}$ is the local velocity of the geometric center of the aerostat or payload, relative to the surrounding air. The variation in drag coefficient of a spherical object from 0.4 to 0.15 , depending on the Reynolds number of the flow, ${ }^{15}$ was included. Once the drag force is calculated, it is decomposed into its inertial frame components for later incorporation into the motion equations.

The added mass of the aerostat and payload were included in the model since they are large. These were calculated as half the displaced air mass of the corresponding spheres. ${ }^{16}$

\section{Assembly of the Motion Equations}

Once all the internal and external forces described above have been expressed in the common inertial frame, the equations governing the motion of each node can be written as

$$
\begin{aligned}
\mathbf{M}^{i} \ddot{\mathbf{r}}^{i} & =\left(\mathbf{T}^{i+1}+\mathbf{P}^{i+1}\right)-\left(\mathbf{T}^{i}+\mathbf{P}^{i}\right) \\
& +\frac{1}{2}\left(\mathbf{D}^{i}+\mathbf{D}^{i+1}+m_{c}^{i} \mathbf{g}+m_{c}^{i+1} \mathbf{g}\right)
\end{aligned}
$$

where $\mathbf{T}^{i}$ and $\mathbf{P}^{i}$ are the elastic and damping force vectors generated in the $i$-th element; $\mathbf{D}^{i}$ is the aerodynamic drag force vector on the $i$-th element; $m_{c}{ }_{c} \mathbf{g}$ is the gravitational force acting on the $i$-th element. The drag, weight and buoyancy of the payload and aerostat are simply added to the appropriate cable nodes (at the lower and upper ends of the leash, respectively). All the node equations are then assembled and integrated simultaneously for $\mathbf{r}^{i}$ the position of node $i$ in inertial space.

\section{Mean Wind and Turbulence Model}

A wind model was incorporated in order to determine its effect on the tethered aerostat system. It consists of a height-dependent mean wind profile on which are superimposed turbulent gusts which also vary with height. The mean wind $U$ at height $h$ was represented by a power law profile ${ }^{17}$ representing the earth's boundary layer

$$
U=U_{g}\left(\frac{h}{h_{g}}\right)^{\alpha}
$$

where the power law exponent $\alpha=0.19$ was used to represent conditions in rural areas. A gradient height $h_{g}$ $=500 \mathrm{~m}$ was used, at which the mean wind reaches its full speed of $U_{g}$.

Turbulent gusts were superimposed on this mean wind. These were generated with the desired gust statistical properties, ${ }^{17}$ including turbulence intensity, scale length and spectra. The turbulence intensities $\sigma_{\mathrm{u}}, \sigma_{\mathrm{v}}$, and $\sigma_{\mathrm{w}}$ in the three orthogonal directions were

$$
\begin{aligned}
& \frac{\sigma_{u}}{U}=\left\{\begin{array}{cc}
0.1 & h \geq 300 \mathrm{~m}
\end{array}\right. \\
& \frac{\sigma_{v}}{\sigma_{u}}=\{\quad 1 \quad h \geq 300 \mathrm{~m} \\
& \frac{\sigma_{u}}{\sigma_{u}}=\{\quad 0.7+h / 1000 \quad h<300 \mathrm{~m} \\
& \begin{array}{ccc}
\sigma_{w} & 1 & h \geq 300 \mathrm{~m} \\
\sigma_{u} & =.25+h / 400 & h<300 \mathrm{~m}
\end{array}
\end{aligned}
$$

while the three corresponding scale lengths $L_{u}, L_{v}$, and $L_{w}$ were

$$
\begin{aligned}
& L_{u}=280\left(\frac{h}{h_{g}}\right)^{0.35}, \quad L_{v}=140\left(\frac{h}{h_{g}}\right)^{0.48} \\
& L_{w}=\left\{\begin{array}{lc}
0.35 h & h \leq 400 \mathrm{~m} \\
140 & h>400 \mathrm{~m}
\end{array}\right.
\end{aligned}
$$

and finally, the three corresponding spectra $\Phi_{\mathrm{u}}, \Phi_{\mathrm{v}}$, and $\Phi_{\mathrm{w}}$ are taken from a von Kàrmàn model ${ }^{17}$ with

$$
\begin{aligned}
& \Phi_{u}(\Omega)=\left(\frac{\sigma_{u}}{U}\right)^{2} U^{2} \frac{L_{u}}{\pi} \cdot \frac{1}{\left[1+\left(a L_{u} \Omega\right)^{2}\right]^{5 / 6}} \\
& \Phi_{v}(\Omega)=\left(\frac{\sigma_{v}}{U}\right)^{2} U^{2} \frac{L_{v}}{2 \pi} \cdot \frac{1+\frac{8}{3}\left(a L_{v} \Omega\right)^{2}}{\left[1+\left(a L_{v} \Omega\right)^{2}\right]^{11 / 16}} \\
& \Phi_{w}(\Omega)=\left(\frac{\sigma_{w}}{U}\right)^{2} U^{2} \frac{L_{w}}{2 \pi} \cdot \frac{1+\frac{8}{3}\left(a L_{w} \Omega\right)^{2}}{\left[1+\left(a L_{w} \Omega\right)^{2}\right]^{11 / 16}}
\end{aligned}
$$

where $\Omega$ is the wave number and $a=1.339$. The following procedure was adopted to generate each of the three gust components

1. Discretize the wave number axis of the spectrum $\Phi_{j}$ into $N=50$ intervals, each of width $\Delta \Omega_{i}=1.2 \Delta \Omega_{i-1}$ $i=1, \ldots, N$, with $\Omega_{0}=0.0001$ and $\Delta \Omega_{0}=0.0001$

2. Randomly choose a wave number $\Omega_{i}$ within each interval.

3. Randomly choose a phase angle $\varphi_{i}$ from 0 to $2 \pi$ for each component.

4. Evaluate $\Phi_{j}\left(\Omega_{i}\right) / U^{2}$

5. Evaluate $A_{i} / U=\left(2 \Phi_{j} \Delta \Omega_{i} / U^{2}\right)^{1 / 2}$

The above steps are all implemented off-line. The last step must be implemented during the dynamics simulation and is a function of position and time.

6. Evaluate the gust speed: 


$$
v_{g}=\sum_{i=1}^{N} \frac{A_{i}}{U} U \cos \left(\Omega_{i}(x-t U)+\varphi_{i}\right)
$$

where $x$ and $t$ are the position along the mean wind direction and time, respectively. The above equation implies the use of a frozen-field approximation $^{17}$ to relate temporal and spatial gust correlations.

It is expected that the above model will incorporate the dominant features of the turbulence statistics, though it does neglect the spatial correlations in gusts in the directions perpendicular to the mean wind velocity.

\section{Winch Controllers}

In this work, straightforward PID controllers were implemented since the intention was primarily to perform an initial comparison of the uncontrolled and controlled systems. The controller on each winch is independent of the other two winch controllers and operates on the position of the payload, which we presume to be accurately measured. A planar representation of the geometry is shown in Figure 5.

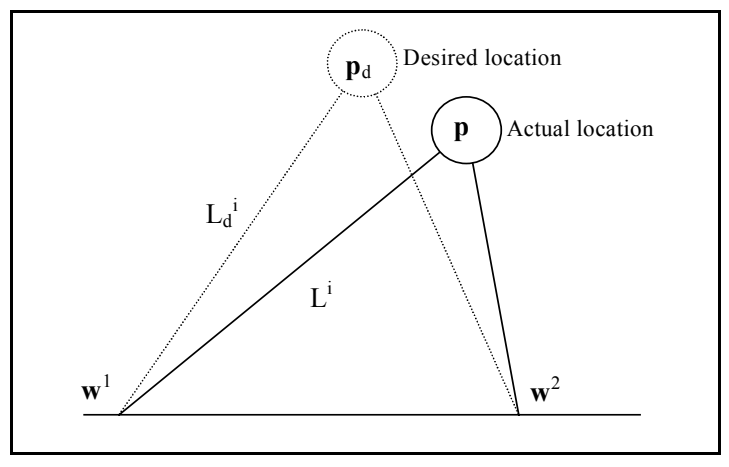

Figure 5. Control Terminology

The desired location of the payload is at $\mathbf{p}_{d}=\left[\begin{array}{lll}x_{d} & y_{d} & z_{d}\end{array}\right]^{\mathrm{T}}$ while its actual location is at $\mathbf{p}=[\mathrm{x} \mathrm{y} \mathrm{z}]^{\mathrm{T}}$. The location of the $j$-th winch $(j=1, . ., 3)$ is at $\mathbf{w}^{\mathrm{j}}=\left[\mathrm{x}_{\mathrm{w}}{ }^{\mathrm{j}} \mathrm{y}_{\mathrm{w}}{ }^{\mathrm{j}} \mathrm{z}_{\mathrm{w}}{ }^{\mathrm{j}}\right]^{\mathrm{T}}$. For each winch, we can therefore define the desired and actual distances to the payload as

$$
L_{d}^{j}=\left\|\mathbf{p}_{d}-\mathbf{w}^{j}\right\|, \quad L^{j}=\left\|\mathbf{p}-\mathbf{w}^{j}\right\|
$$

Our winch controller can now operate according to

$l_{u}^{1}=l_{u 0}^{1}-k_{d}\left(\dot{L}_{d}^{j}-\dot{L}^{j}\right)-k_{p}\left(L_{d}^{j}-L^{j}\right)-k_{i} \int\left(L_{d}^{j}-L^{j}\right) d t$

where $l_{u}{ }^{l}$ is the unstretched length of the first (lowermost) element in tether $j$, while $l_{u 0}{ }^{l}$ is its initial length. The motivation behind this approach is that, if the distance from winch $j$ to the payload is correct, then the payload lies on a sphere of radius $L_{d}^{j}$ centered at the winch. If all three payload-winch distances are correct, then the payload lies at the intersection of those spheres which define the correct desired location of the payload in 3-D space.

\section{Numerical Implementation}

The second order differential equations given by (12) must now be solved by numerical integration. Each tether consists of $n_{t}$ elements---and the last of these are connected at the leash---so that there are a total of $3\left(n_{t}+1\right)-2$ nodes in the tether system. The leash consists of an additional $n_{l}$ elements, and, since the lowermost end node is common to the three tethers, a total of $n_{l}$ nodes are added to the system, thus leading to a total of $3\left(n_{t}+1\right)-2+n_{l}$ nodes in the system. Each node is modeled by three second-order ODEs (one for each nodal degree of freedom). However, the lowermost node of each tether has no differential equations associated with it because its motion is prescribed. Furthermore, the aerostat and payload do not add any differential equations since their effect is lumped into the leash end nodes. In order to apply a numerical integration algorithm in standard form, each secondorder ODE is rewritten as two first-order ODEs. This then leads to a total of of $18 n_{t}-12+6 n_{l}$ first-order ODEs to represent the complete system.

The approach taken to solve the system of differential equations is taken from Reference 18. The particular integrator chosen is a fourth order Runge Kutta technique with fixed step size. ${ }^{18}$ This method gives accurate solutions for a broad range of problems, though not necessarily with optimum efficiency. Because the system of differential equations is large (180 first order ODEs for $n_{t}=10$, and $n_{l}=2$ ), and the cable material is stiff $\left(\mathrm{E}=1.7 \times 10^{10} \mathrm{~Pa}\right)$, a small time step had to be used to avoid numerical instabilities and ensure an accurate solution. Accuracy tests were made to ensure that an appropriate time step was used, ultimately leading to a time step of $1 \mathrm{~ms}$.

\section{Numerical Results}

The particular configuration simulated was one in which the payload's desired position was at a fixed point on a hemisphere of radius $\mathrm{R}=500 \mathrm{~m}$ whose center lay on the earth's surface. The winches were each located $1200 \mathrm{~m}$ from the hemisphere's center and configured symmetrically $120^{\circ}$ apart. The desired location of the payload is specified by its zenith $\theta_{z a}$ and azimuth $\theta_{a z}$ angles, shown in Figure 6.

From this geometry, we find that the desired position coordinates of the payload are

$$
\mathbf{p}_{d}=\left[\begin{array}{llll}
R \cos \theta_{a z} \sin \theta_{z a} & R \sin \theta_{a z} \sin \theta_{z a} \quad R \cos \theta_{z a}
\end{array}\right]^{T}
$$




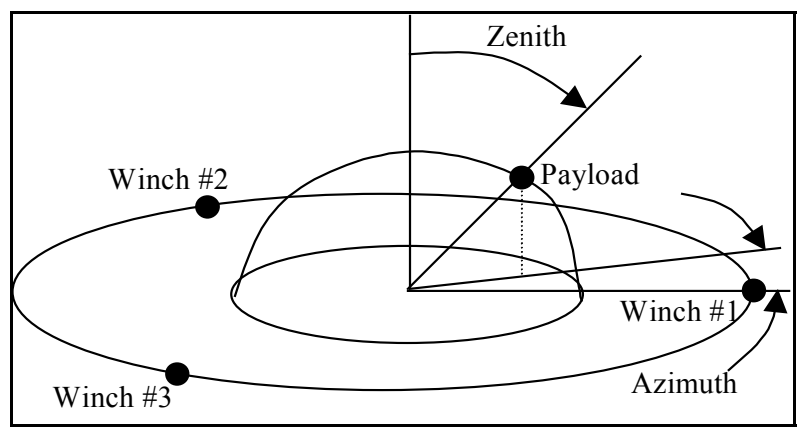

Figure 6. Geometry of Layout

The tether and leash consisted of Samson Spectron rope of $1.85 \mathrm{~cm}$ diameter, with a density of $840 \mathrm{~kg} / \mathrm{m}^{3}$ and a normal drag coefficient of 1.2 . The $500 \mathrm{~kg}$ payload was contained in a sphere of $6 \mathrm{~m}$ diameter, while the spherical aerostat had diameter of $19.7 \mathrm{~m}$, a mass of $610 \mathrm{~kg}$, and a gross buoyancy of $40870 \mathrm{~N}$.

The simulation described above was first validated by comparing its steady-state results against an independently-generated statics simulation. ${ }^{8}$ All results matched within a fraction of a percent, both with and without steady winds (no turbulence).

A range of test cases at different azimuth and zenith angles was then run, for a variety of wind conditions. Two sets of results are shown here to highlight salient features of the system's performance. The first set of results compares the uncontrolled and controlled behavior of the system when $\theta_{z a}=\theta_{a z}=0$ i.e., the desired payload position is directly above the center of the main reflector. The mean wind and turbulence are absent for the first 5 seconds, at which time $U_{g}$ rises to $10 \mathrm{~m} / \mathrm{s}$, directed along zero azimuth. The payload motion and winch tensions are shown in Figures 7 and 8 , respectively, with and without winch control. The motion is plotted as components in and out of the focal plane (the plane locally tangent to the $500 \mathrm{~m}$ hemisphere at the desired aerostat location) because only those errors in the focal plane are of primary importance. When $\theta_{z a}=\theta_{a z}=0$, the focal plane is horizontal and the out-of-plane motion is purely vertical. The uncontrolled case makes apparent that the system responds little to high-frequency gusts and acts as a natural low-pass filter due to its huge scale. However, the error in the focal plane peaking at $1.5 \mathrm{~m}$ is unacceptable (though detailed ranges of acceptable motion have not yet been fully defined). When winch control is applied with $k_{d}=2 \mathrm{~s}, k_{p}=5, k_{\mathrm{i}}=5 \mathrm{~s}^{-1}$, the peak focal plane error is reduced to less than $5 \mathrm{~cm}$.

The second test case shows the performance of the system for $\theta_{z a}=60^{\circ}, \theta_{a z}=0$ primarily to highlight the degradation in system behavior at large zenith angles. The motion errors and winch tensions are shown in
Figs. 9 and 10, respectively. It was found that at large zenith angles, the controller gains had to be reduced to avoid instabilities, indicating that the system is inherently less stable. The motion of the uncontrolled system at large zenith angles supports this interpretation. When compared to the preceding test case, the system oscillations appear much less damped. For the large zenith angles, it was also difficult to find a stable initial condition, and this is indicated by the nonzero motion of the system at $\mathrm{t}=0$. The controller, with $k_{d}=4 \mathrm{~s}, k_{p}=1$, and $k_{\mathrm{i}}=1 \mathrm{~s}^{-1}$, reduces the motion of the system, but not as significantly as in the first test case.

Other test cases, not shown here, revealed that the system is not very sensitive to the azimuth angle of the payload, and somewhat sensitive to the mean wind direction. Based on these findings, we expect that a long-term control solution will have to be adaptive in nature and change its characteristics at least with the payload zenith angle. It is also likely that a model-based controller could be applied to good advantage.

\section{Conclusions}

The computer simulation described here provides an efficient and cost effective method of determining the effects of different wind conditions and aerostat configurations on the tethered aerostat system described. The key findings of the study are: (a) the system is relatively insensitive to turbulent gusts and only responds to the lowest frequency gusts; (b) the system is significantly less well behaved (i.e. less stable) at large zenith angles, and (c) simple controllers developed in the course of this short study appear to be capable of reducing the motion response of the confluence point within a few centimeters of its desired location.

Future work will include incorporation of a streamlined aerostat model, incorporation of more than three tethers, improvement of the turbulence model, and an investigation of adaptive model-based controllers. Experimental validation at model scale would also be advantageous to confirm the computer model results.

\section{Acknowledgements}

This work was supported financially by the Natural Sciences and Engineering Research Council and the National Research Council.

\section{$\underline{\text { References }}$}

[1] 'Memorandum of an Agreement to Cooperate in a Technology Study Program Leading to a Future Very Large Radio Telescope', signed by participants from Australia, Canada, China, India, the Netherlands, the U.S.A. http://www.nfra.nl/skai/memorandum.htm 
[2] R. van de Weygaert and T. S. van Albada, 1996, 'New Challenges for Cosmology', The Westerbork Observatory, Continuing Adventure in Radio Astronomy, Eds. E. Raimond and R. Genee, Astrophysics and Space Science Library Volume 207, pp. 225--259.

[3] T. H. Legg, 1998, 'A Proposed New Design for a Large Radio Telescope', Astronomy and Astrophysics Supplement, v.130, p.369-379.

[4] S. P. Jones and J.A. Krausman, 1982, 'Nonlinear Dynamic Simulation of a Tethered Aerostat', AIAA Journal of Aircraft, Vol. 19, No. 8, pp. 679-686.

[5] S. P. Jones, 1987, 'Nonlinear Dynamic Simulation of a Moored Aerostat', AIAA Paper 87-2505.

[6] S. P. Jones and J. D. DeLaurier, 1983, 'Aerodynamic Estimation Techniques for Aerostats and Airships, AIAA Journal of Aircraft, Vol. 20, No. 2, pp. $120-126$.

[7] S. Badesha and S. P. Jones, 1993, 'Aerodynamics of the TCOM 71M Aerostat', AIAA Paper 93-4036-CP.

[8] B. Veidt and P. Dewdney, 1996, 'Steady-State Stability Analysis of a Triple Tethered Balloon Platform', DRAO Future Radio Facilities Report \#1.

[9] F. R. Driscoll and M. Nahon, 1996, 'Mathematical Modeling and Simulation of a Moored Buoy System', Proceedings of Oceans'96, pp. 517-523.

[10] B. Buckham, M. Nahon and M. Seto, 'ThreeDimensional Dynamics Simulation of a Towed Underwater Vehicle, Proceedings of OMAE'99, the $18^{\text {th }}$ International Conference on Offshore Mechanics and Arctic Engineering.

[11] F. R. Driscoll, 1999, 'Dynamics of a Vertically Tethered Marine Platform', PhD. Thesis, University of Victoria.

[12] R. Folb and J. J. Nelligan, 1983, 'Hydrodynamic Loading on Armored Towcables', David W. Taylor Navel Ship Research and Development Center, DTNSRDC-82/116.

[13] M. J. Casarella, 1970, 'Survey of Investigations on the Configuration and Motion of Cable Systems Under Hydrodynamic Loading', Marine Technology Society Journal, Vol. 4, No. 4, pp. 27-44.

[14] Y. Choo, 1971, 'Hydrodynamic Resistance of Towed Cables', Journal of Hydronautics, Vol. 5, No. 4, pp. 126-131.

[15] B. W. McCormick, 1995, Aerodynamics, Aeronautics and Flight Mechanics, John Wiley \& Sons.

[16] J. N. Newman, 1989, Marine Hydrodynamics, MIT Press.

[17] B. Etkin, 1972, Dynamics of Atmospheric Flight, John Wiley \& Sons.
[18] W. H. Press, et al., 1992, Numerical Recipes in C, Cambridge University Press.
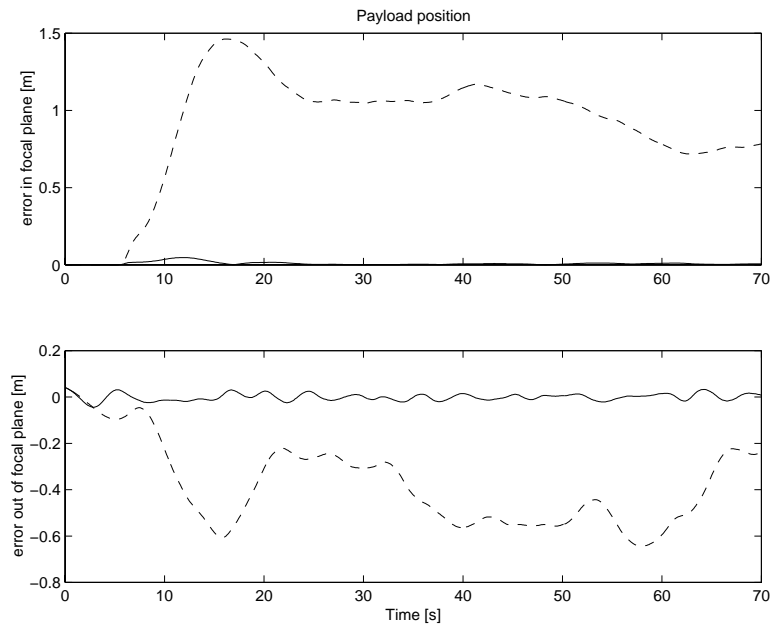

Figure 7. Payload Motion for $\theta_{z a}=\theta_{a z}=0 \mathrm{With}$ Control (solid line) and Without Control (dashed line)
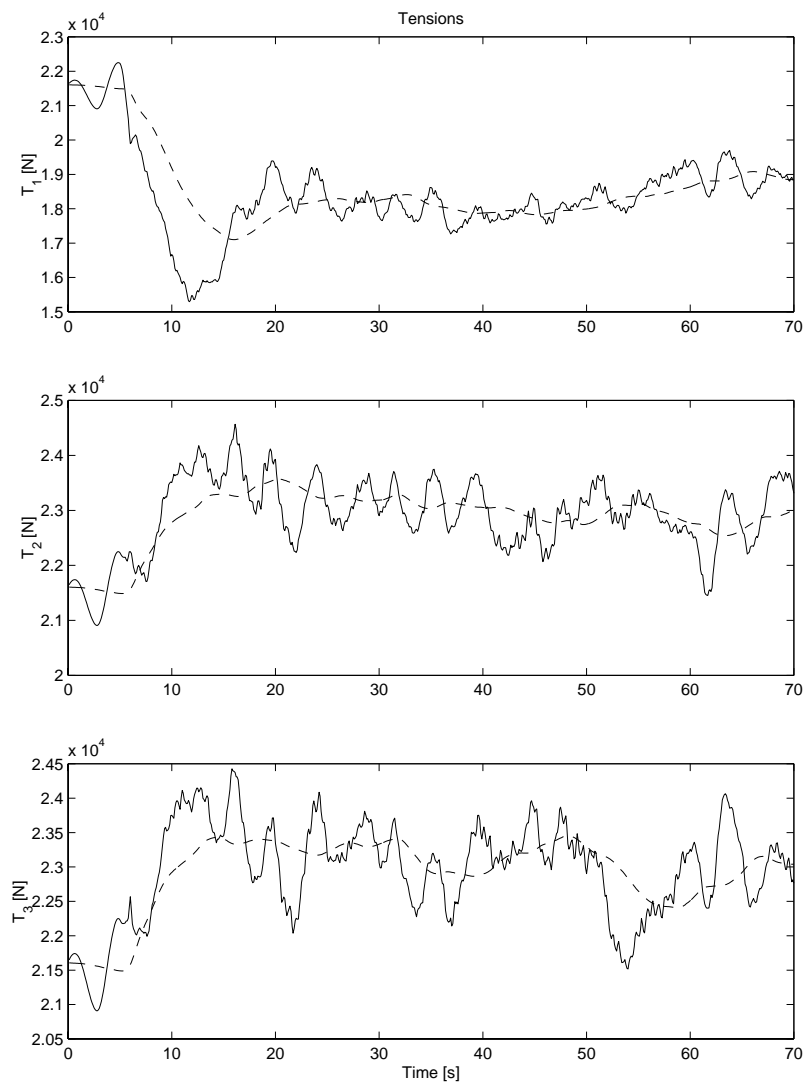

Figure 8. Winch Tensions for $\theta_{z a}=\theta_{a z}=0$ With Control (solid line) and Without Control (dashed line) 

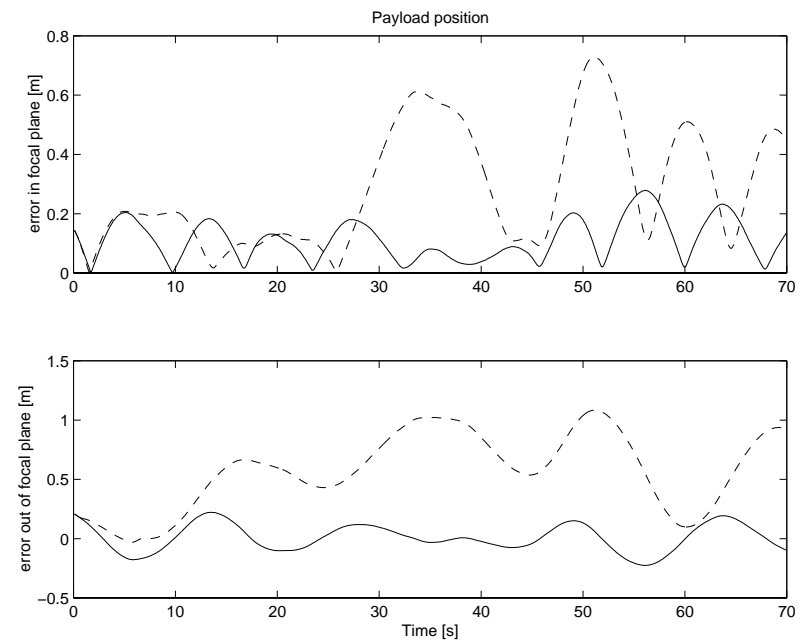

Figure 9. Payload Motion for $\theta_{z a}=60^{\circ}, \theta_{a z}=0$ With Control (solid line) and Without Control (dashed line)
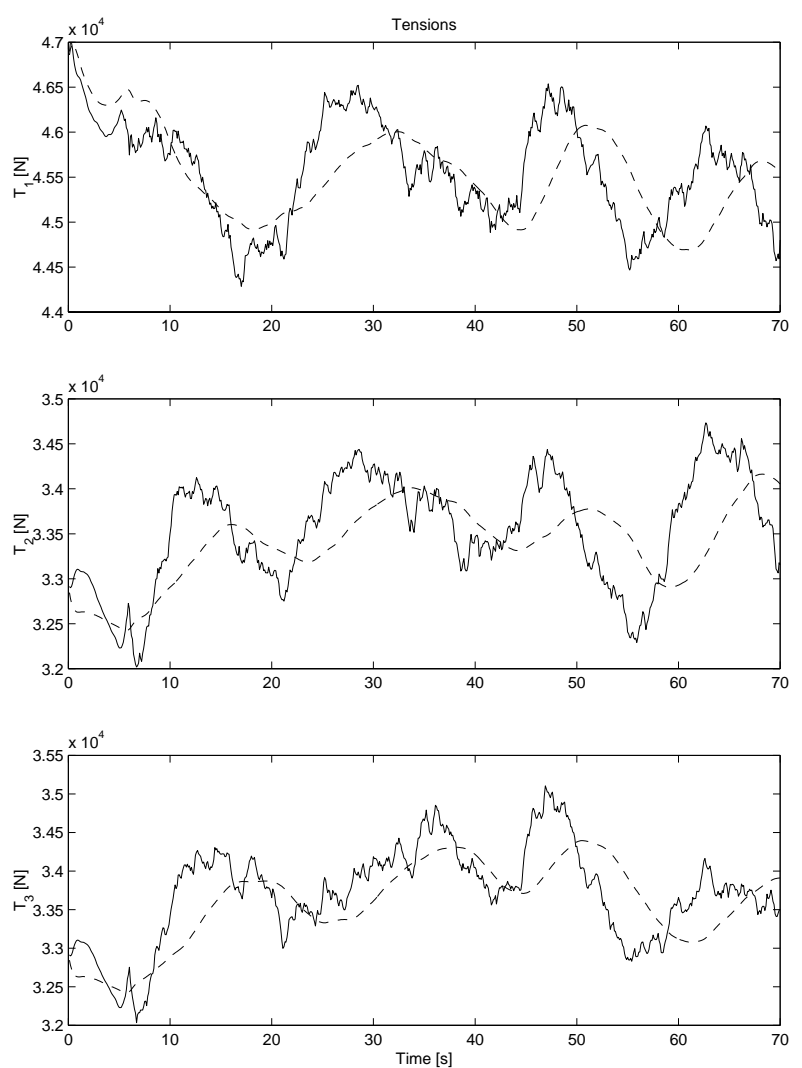

Figure 10. Winch Tensions for $\theta_{z a}=60^{\circ}, \theta_{a z}=0$ With Control (solid line) and Without Control (dashed line) 